\title{
Development of a protocol for guidance in the pediatric nursing practicum in South Korea: a methodology study
}

\author{
Hyun Young $\mathrm{Koo}^{1}$, Bo Ryeong Lee ${ }^{2}$ \\ ${ }^{1}$ Professor, College of Nursing • Research Institute of Nursing Science, Daegu Catholic University, Daegu; ${ }^{2}$ Graduate Student, College of Nursing, Daegu Catholic \\ University, Daegu, Korea
}

\begin{abstract}
Purpose: Practical training in pediatric nursing gives students the opportunity to apply nursing knowledge to children in a realistic clinical context. Clinical practice faculty $(\mathrm{CPF})$ and clinical nursing instructor (CNI) have played important roles in the pediatric nursing practicum. This study was conducted to develop a protocol to guide clinical practicum in pediatric nursing. Methods: A service design methodology was employed between August 2020 and May 2021 at four universities and four hospitals in South Korea. The participants were five $\mathrm{CPFs}$, five CNIs, five nursing college graduates, and 60 nursing students. The service design process had four phases: discovery, definition, development, and delivery. Data were collected through self-report questionnaires, in-depth interviews, and observations. The data were analyzed using content analysis and descriptive statistics. Results: The participants reported needs for providing concrete guidance and explanation, nursing practice experience, and a link between school and the clinical field. A protocol was developed to fulfill the participants' needs. The protocol comprised detailed information, teaching methodology, and partnership to guide students in the pediatric nursing practicum. Conclusion: The protocol developed in this study can be used to provide guidance for students' clinical practice in the field of pediatric nursing.
\end{abstract}

Key words: Practice guideline; Pediatric nursing; Students, nursing

\section{Corresponding author Hyun Young Koo}

College of Nursing · Research Institute of Nursing Science, Daegu Catholic

University, 33 Duryugongwon-ro, 17 gil,

Nam-gu, Daegu 42472, Korea

TEL: +82-53-650-4829

FAX: $+82-53-650-4392$

E-MAIL: hykoo@cu.ac.kr

Received Sep 24, 2021

Revised Oct 21, 2021

Accepted Nov 8, 2021

\section{INTRODUCTION}

In order for nursing students to become professional nurses, they must practice applying the nursing knowledge from their theoretical education to the clinical field by identifying and solving nursing problems. Theoretical and practical education is provided for various nursing specialty areas, and nursing students learn about the nursing processes involved in health assessment, promotion, and recovery for children through clinical practice [1]. However, the coronavirus disease 2019 (COVID-19) pandemic has limited the ability of nursing students to participate in clinical practice in the pediatric ward in order to protect vulnerable pediatric patients and increased nurses' workload, reducing their capacity to teach and supervise students [2].

Nursing practice is integral for improving nursing compe- tency. Therefore, the Korean Accreditation Board of Nursing Education (KABONE) [3] requires more than 1,000 hours of clinical practice to achieve the documented learning goals, and a clinical nursing instructor (CNI) affiliated with the practicum institution and a university-affiliated clinical practice faculty $(\mathrm{CPF})$ with professional competency and clinical experience must be present to instruct students during clinical practice.

Despite the importance of clinical practice, nurses in the clinical field instruct students without prior experience as educators [4], and training and preparation for practicum instruction are insufficient. CPFs and CNIs lack information about each other [5] and experience difficulties establishing collaborative relationships [6]. To operate clinical practice efficiently, a standardized practicum instruction guideline is necessary [4], and interactions and collaboration between 
schools and the clinical site are emphasized [5]. To enhance nursing students' experiences of pediatric nursing practice, $\mathrm{CPFs}$ and CNIs should prepare to instruct students during the pediatric ward practicum in a way that reflects the learning goals and considers the characteristics of pediatric nursing.

$\mathrm{CPFs}$ and CNIs are important contributors to nursing students' professional training, which combines nursing knowledge and skills. In South Korea, CPFs are faculty, part-time lecturers, and teaching assistants affiliated with universities who oversee the instruction of students' clinical practice [3]. CPFs play a very important role in the efficient operation of clinical practice, and students viewed their clinical practice to be adequate when they perceived the instruction by CPFs to be efficient [7]. Recently, in order to secure excellent instructors for clinical practice, nursing specialists or managers who are in charge of nursing practice in the field have been assigned to CPF. Prior notice and ongoing support are necessary for them to fulfill their roles in student education and instruction $[8,9]$.

In South Korea, CNIs practice at the affiliated practicum institution and include head nurses, unit managers, and preceptors appointed by the university for instruction in clinical field practice. They must be qualified with a bachelor's degree or higher and 3 or more years of clinical experience [3]. CNIs influence the improvement of practice competencies of nursing students by providing students with direct experiences of work capabilities, interpersonal relationships, and communication [10] and teaching them clinical decision-making and nursing skills [9]. However, CNIs experience difficulties due to excessive workload, allow students only to observe (rather than engage in hands-on practice) due to legal and ethical concerns, and feel limitations in practicum instruction [11]. In particular, due to the COVID-19 pandemic, many nursing students have been restricted from entering the hospital, not allocated to their practicum departments according to the clinical practice plans, and not given the opportunity to practice nursing skills [2].

Therefore, detailed and practical guidelines should be provided for CPFs and CNIs to provide effective instruction in the pediatric practicum during the COVID-19 pandemic. In order for the practicum instruction guidelines to be used effectively, the guidelines should sufficiently reflect the experiences of CPFs and CNIs, who are the users, as well as the experiences of nursing students, and content based on their needs and issues should be provided. The service design method can be used for user-centered protocol development; this framework creates results by meeting users' needs, solving problems, and considering users' overall context [12].

As a service design method, the double diamond process [13] finds users' latent needs, derives ideas to fulfill those needs, and actualizes the ideas in the discovery, definition, development, and delivery phases. According to the double diamond process [13], data are collected from a literature review, interviews, and surveys in the discovery phase to identify users' needs and issues that require improvement. In the definition phase, the collected data are systematically integrated to derive a problem using brainstorming or mind mapping, and the solution to the problem is expanded into an idea in the development phase. The idea is condensed into a detailed protocol or blueprint in the delivery phase. The double diamond process [14] expands, organizes, and derives ideas through expansion and convergence. The service design method is useful to identify users' needs, in addition to creating specific output to meet those needs. Thus, the service design can be used to provide a guideline for instruction in the pediatric nursing practicum by developing a protocol for $\mathrm{CPFs}$ and $\mathrm{CNIs}$.

\section{Purpose}

This study aimed to develop a protocol for pediatric practicum instruction, provide guidelines for pediatric nursing practicum instruction to CPFs and CNIs, and improve practice education and instruction methods. The goal of this study was to use the service design methodology [13] to identify the needs and problems of CPFs, CNIs, and nursing students to develop a detailed and practical pediatric nursing practicum instruction protocol.

\section{METHODS}

Ethics statement: This study was approved by the institutional review board of Daegu Catholic University (No. CUIRB-2020-0007). Informed consent was obtained from all participants.

\section{Study Design}

This is a methodological study that developed an instruction protocol for the pediatric nursing practicum instruction by applying the double diamond process [13], which is a service design method.

\section{Participants and Setting}

Five CPFs, five CNIs, five nursing college graduates, and 60 senior nursing students who had experienced the pediatric ward practicum (40 in the discovery stage, 20 in the delivery stage) were identified through convenience sampling from four nursing schools, two general hospitals, and two pediatric 
hospitals in two large cities and two mid-sized cities in South Korea. The number of participants was determined with reference to the fact that service design studies conducted to develop guidelines and education programs collected data from 50-74 users [15,16]. Data were collected from five CPFs, five CNIs, five graduates of nursing school, and 40 senior nursing students in the discovery phase. Data were collected from two $\mathrm{CPFs}$ and two CNIs who had already provided data in the discovery phase and 20 different senior nursing students. No participants in the study dropped out.

\section{Service Design and Data Collection}

\section{1) Discovery}

In order to identify users' needs and problems that require improvement, the literature regarding pediatric nursing practicum education and instruction was reviewed. Surveys and interviews were conducted with users (CPFs, CNIs, nursing school graduates, and senior students), and the circumstances of instruction in the pediatric nursing practicum were observed and analyzed.

The survey and interviews occurred from August 27, 2020 to February 15, 2021; throughout this period, the COVID-19 pandemic was ongoing in South Korea. Pediatric ward practicum education, instructional experience, and areas for improvement were examined using open-ended questions with five $\mathrm{CPFs}$, five CNIs, five nursing school graduates, and 40 seniors who had experienced pediatric ward practicum who agreed to participate in the study. The open- ended questions were constructed based on the related literature and the researchers' experience with the goal of deriving the needs and areas for improvement in pediatric nursing practicum education and instruction. The questions included "Please describe your pediatric practicum education and instruction experience", "Please tell us about what you think is important in pediatric practicum education and instruction", and "Please tell us what you wish would be improved in pediatric practicum education and instruction". After reviewing the responses in narrative form, when follow-up questions were needed, an interview was conducted at a time and location convenient for the participants. Phone calls were also made to prevent the spread of COVID-19. Among the survey respondents, two $\mathrm{CPFs}$, one $\mathrm{CNI}$, and eight senior students were interviewed either in-person or on the phone. The interviews were 20-30 minutes long, and the interviews were transcribed to be analyzed together with the narrative survey responses.

One researcher visited a pediatric ward of a teaching hospital where clinical practicum of nursing students was taking place during the period when the survey and interviews were being conducted. The researcher observed the location and structure of a pediatric ward, students' paths and activities in the ward, and the practicum instruction. The researcher did not participate in any activities with students during the 1-hour observation (2 PM to 3 PM), but if needed, she could ask a student for clarification. Observations about the structure of the pediatric ward, the space for students, and students' specific activities in the ward were objectively recorded using field sketches and memos.

\section{2) Definition}

Through brainstorming, researchers freely suggested various ideas about user needs. The problems that required improvement were displayed as images through mind mapping. The collected data were systematically integrated and reduced to derive a problem.

\section{3) Development}

Solutions to the derived problem were expanded into an idea, which was organized into detailed content to develop a pediatric nursing practicum instruction protocol.

\section{4) Delivery}

Surveys and interviews to receive feedback on the developed protocol were conducted from March 15, 2021 to May 20, 2021 when the COVID-19 pandemic was ongoing in South Korea. The developed protocol was reviewed two CPFs and two CNIs who had already provided data in the delivery phase and 20 different senior nursing students. Feedback on the organization and content of items was sought, and the protocol was finalized after revisions based on the feedback.

\section{Ethical Considerations}

For the ethical protection of participants, the study was conducted after approval (CUIRB-2020-0007) from Daegu Catholic University Institutional Review Board (IRB). The study aim, methods, and procedure were explained to potential participants, and they were informed that they did not have to participate if they did not wish to and could discontinue participation at any time and for any reason. They were also informed that anonymity and data privacy will be honored and that the data will be discarded after the study. Participants were included in the study only after they decided to participate in the study voluntarily and provided written consent. A small gift was provided. Data collection and analysis followed the principles of research ethics.

\section{Data Analysis}

Narrative responses and interview data collected from par- 
ticipants in the discovery phase were analyzed using the inductive approach of qualitative content analysis [17]. In the preparation stage, the content was analyzed after reading the narrative responses and interview transcripts multiple times to grasp the overall data. In the organizing stage, meaningful statements were extracted and categorized by similar characteristics. The observational data were used to review detailed factors of derived themes. In the reporting stage, categories were suggested.

The general characteristics of participants and the survey data from the delivery phase were analyzed using descriptive statistics.

\section{RESULTS}

\section{General Characteristics of Subjects}

The CPFs were all women, with an average age of 43.8 years $(\mathrm{SD}=4.0)$, and their average length of practicum instruction was 11.4 years $(S D=3.8)$. The $C N I s$ were all women, with an average age of 44.8 years ( $S D=10.3)$, and their average length of practicum instruction was 17.7 years $(S D=11.4)$. The nursing school graduates included four women and one man. Their average age was 24.0 years $(S D=1.6)$, and their average length of work after graduation was 1.3 years $(S D=0.7)$. The nursing students were all seniors, including 55 women and five men. Their average age was 22.3 years $(S D=1.2)$.

\section{Discovery}

\section{1) Literature review}

According to a recent study [10], several professional competencies-including theoretical and practical knowledge and skills, the ability to form interpersonal relationships, and an approachable personality-are needed to provide effective clinical practice instruction for nursing students. CPFs and CNIs who were newly assigned to practicum instruction expressed the desire to be informed about clinical practice regulations, the status of completion of theoretical training related to clinical practice, and student evaluations [18] and found manuals with detailed and clear information about the school and the practicum field to be useful [4]. For practicum instruction, beyond basic information about the operation of practicum education, there is also a need for knowledge and skills regarding teaching and learning methods [18]. To improve the pediatric nursing practicum, various simulation techniques have been attempted $[19,20]$.

2) Needs for guidance in the pediatric nursing practicum

(1) Needs of CPFs
Concrete guidance and explanation: $\mathrm{CPFs}$ stated that it is necessary to introduce the practicum field sites during orientation.

The CNI usually explains the ward structure and precautions in the orientation. We need a more practical orientation. For example, introducing inpatients will help students adapt to practice. (CPF 5)

Providing nursing practice experience: CPFs felt the need for problem-based learning (PBL) and simulation practice since the direct nursing care provided by students was restricted for the protection of vulnerable pediatric patients due to the COVID-19 pandemic, and the practicum centered on observation.

Even for the seniors, it is difficult to obtain meaningful data from the patient and decide the appropriate nursing diagnosis. So, it is necessary to develop students' ability to think on their own through PBL. These days, the number of hospitalized children due to COVID-19 is small, and students cannot do anything with children, so a variety of topics should be included in the practicum in school. (CPF 1)

Link between school and the clinical field: CPFs stated that it is necessary to have prior discussions about the process of clinical practice with CNIs, and collaborate with CNIs through regular management meetings or workshops.

The important thing is to form a learning atmosphere where students learn directly from nurses, so communication through meetings with head nurses is necessary. (CPF 3)

(2) Needs of CNIs

Concrete guidance and explanation: CNIs desired to receive detailed instructions from academic institutions on the cases that students should learn about and how they should do so.

I don't know what students know, and they can't do anything here. Therefore, I think that students don't know anything. What should I teach the students? I don't even know what to teach them among the many possible options. I want the school to let me know students' needs and what to teach them. (CNI 5)

Providing nursing practice experience: CNIs found it difficult to instruct students due to their busy schedules. Parents 
of children sometimes dislike it when students approach, and CNIs recognized that the nursing care that can be provided by students is practically limited. CNIs expressed that supplementation is necessary since the COVID-19 pandemic has made students' pediatric ward practicum difficult.

There is limited work that students can do directly in the pediatric ward. This is because various diagnostic tests and treatments are often conducted in separate spaces, and guardians often take an exclusionary posture. It has become more difficult because of COVID-19; therefore, supplementation in school is necessary. (CNI 2)

Link between school and the clinical field: CNIs also wished to communicate and collaborate with CPFs and stated that it is necessary to recognize students' right to be educated.

In fact, the educational direction required by the hospital and the educational topics required by the school are different, so it would be better for faculty and nurses to develop the practice guide together. If we develop it together, students would learn a lot, and nurses would instruct more actively. (CNI 1)

(3) Needs of nursing graduates

Concrete guidance and explanation: Graduates of nursing school wished that the pediatric ward administration would tell students specifically what they can and cannot do and that nurses would explain their nursing activities to students when they cared for children.

According to my experience, students want to be instructed on what they should learn, what they can do, and what they shouldn't do in unfamiliar practice sites. (nursing graduate 3 )

Providing nursing practice experience: Graduates expressed that students should prepare for the practicum by studying children's developmental characteristics and main diseases, as well as the corresponding examinations and treatments in school. Furthermore, graduates stated that nursing activities that cannot be done in the pediatric ward practicum should be supplemented using simulations.

There is a limit to what students can experience during the field practicum. Students can make up for these limits through the in-school practicum. The subjects of pediatric simulations are coping with febrile seizures or performing pediatric cardiopulmonary resuscitation. (nursing graduate 4)
Link between school and the clinical field: Graduates perceived nurses who worked skillfully in the pediatric wards as their role models as professional medical providers.

When the nurse performed an intravenous injection, I couldn't see the blood vessels well, and the baby moved and cried. However, two nurses soothed the baby and carried out the injection. They looked professional. I thought I should be a competent nurse like them. (nursing graduate 2)

(4) Needs of nursing students

Concrete guidance and explanation: Nursing school students wanted a detailed orientation from CNIs and kind explanations from nurses. Specifically, students wanted CNIs to explain the structure of the pediatric ward, examination, treatments, nursing schedules, nurses' duties, and instructions for using electronic medical records on the first day of the practicum. Students wanted nurses to provide explanations while caring for children.

It would be nice to go around every corner of the ward and tell us in detail where and what things are. Also, I want nurses to tell us what they're doing when they do something or write something down. (nursing student 5)

Providing nursing practice experience: Students regretted that they could not directly provide care to children due to the COVID-19 pandemic and the risk of infection. They expressed the wish to closely observe children's examinations and treatment.

Because of COVID-19, nurses emphasize infection control to students. Therefore, students are restricted from providing care to children. I hope to observe nurses' performance in the pediatric ward. (nursing student 2)

Link between school and the clinical field: Students wanted to have similar experiences to actual clinical situations through clinical performance examinations (CPXs) in school, to receive sufficient feedback from CPFs, and to have more frequent simulation practice that reflects clinical situations.

It is helpful to provide simulation training before the clinical practice. It was good to have similar experiences to actual clinical situations in school. (nursing student 1 )

\section{3) Observation of practicum instruction}

When a researcher visited the pediatric ward where clinical practice was being conducted, three students were gathered 
in the hallway, and one student was in the clinical examination room with a child. Nurses were busy doing their jobs in the pediatric ward. One of the students who was gathered in the hallway went into the ward to measure a child's vital signs, and the student who was in the clinical examination room returned to the ward with the child. The student said that she could not observe the examination process and returned after waiting outside The pediatric ward practicum takes place for 5 days with 3-4 students on one team, who observe the nursing activities of the nurse they were assigned to by the CNI each morning before the start of the practicum. However, nurses have to work quickly, so in many cases they work without providing students the opportunity to observe. Caregivers who are particularly sensitive due to the COVID -19 pandemic often dislike students approaching. Therefore, students gather together from time to time without anything to do. Students experience simple and low-risk activities such as measuring children's vital signs and accompanying them to the examination room.

\section{Definitions}

Through brainstorming and mind mapping, participants' needs and requirements were combined and reduced to three areas: "concrete guidance and explanation", "providing nursing practice experience", and "link between school and the clinical field" (Figure 1).
"Concrete guidance and explanation" for CPFs and CNIs refers to providing detailed information about the procedure, characteristics of the practicum department, and nursing activities that can be done by students at the beginning of the pediatric ward practicum and explaining appropriate content in detail during the practicum.

"Providing nursing practice experience" for CPFs and CNIs involves providing simulation experiences similar to actual clinical situations as an alternative given that direct nursing is difficult due to the restrictions in contacting vulnerable pediatric patients and the risk of infection.

"Link between school and the clinical field" for CPFs and CNIs refers to regular communication and collaboration between CPFs from the university and CNIs from the practicum institution to reduce the gap between theory and practice.

\section{Development}

Ways to meet the derived participant needs were expanded into an idea. "Detailed information", "teaching methodology", and "partnership" were composed as items and organized into detailed content to develop a protocol for pediatric nursing practicum instruction (Figure 2).

"Detailed information" refers to detailed and practical information required by $\mathrm{CPFs}$ and $\mathrm{CNI}$ s for pediatric ward practicum instruction and fulfills the "concrete guidance and explanation" need that was derived in the definition phase.

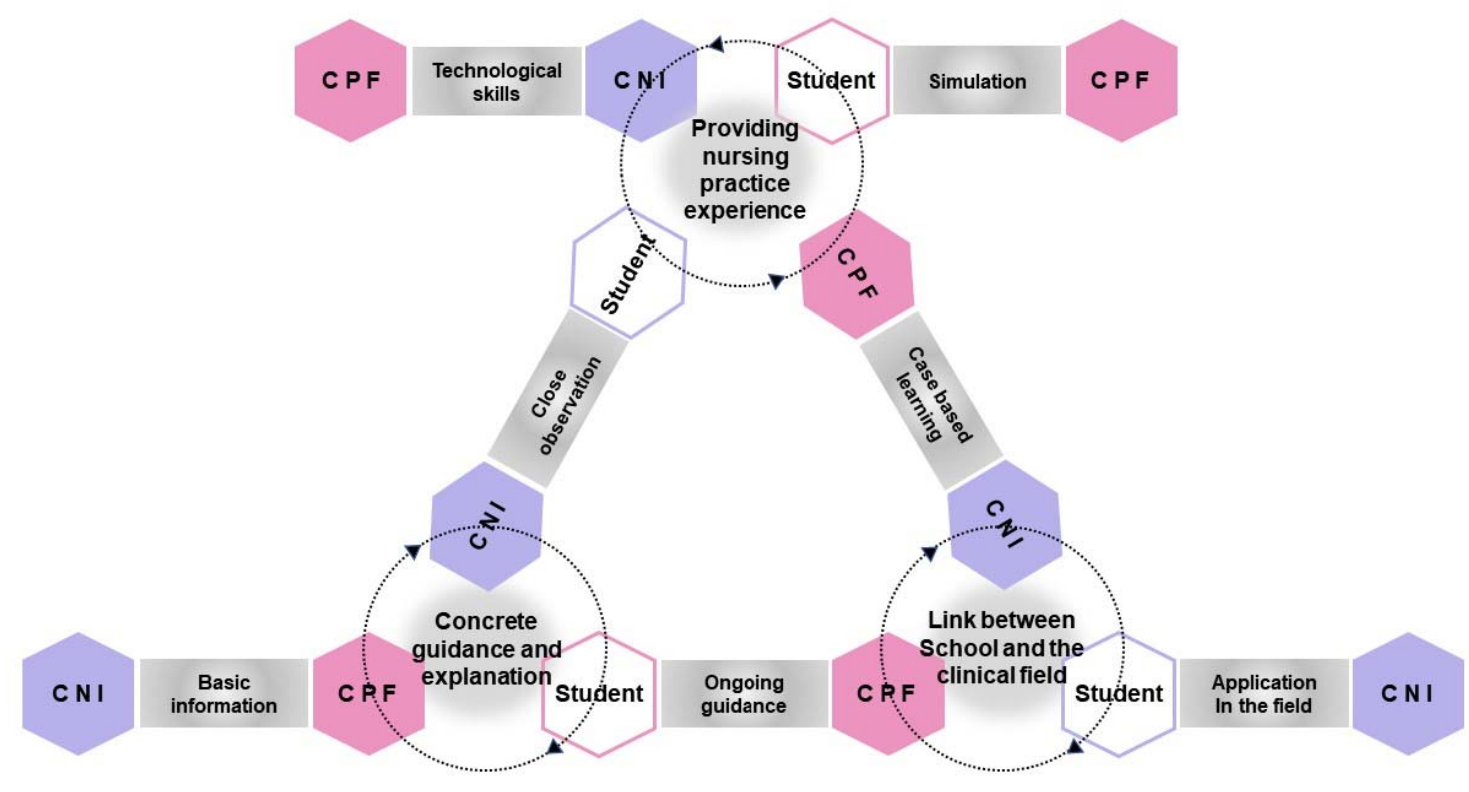

Figure 1. Definition of needs for guidance in the pediatric nursing practicum. $\mathrm{CNI}$, clinical nursing instructor; $\mathrm{CPF}$, clinical practice faculty. 


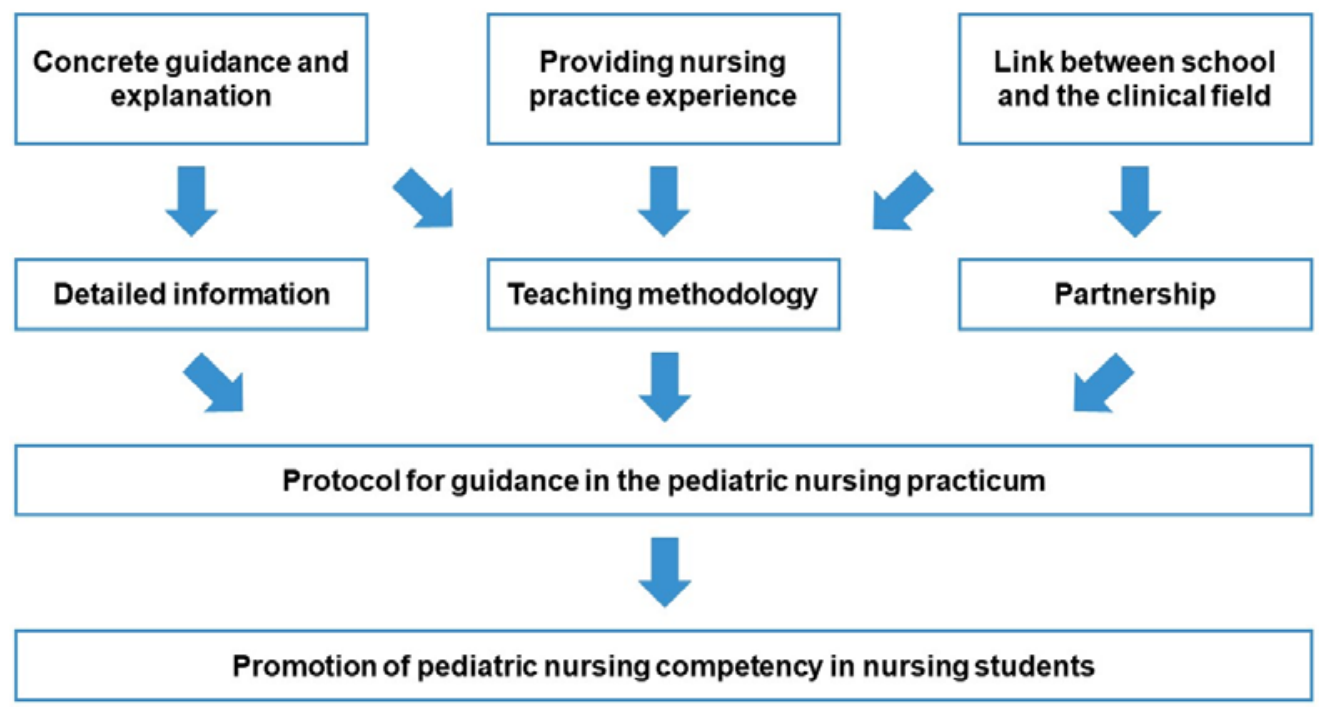

Figure 2. Development of the protocol for guidance in the pediatric nursing practicum.

This item is organized into regulations for operating clinical practice, the role of CPFs and CNIs, curriculum, practicum schedule, student assignment charts, student evaluation criteria and tools, and contact information of schools and practicum institutions.

"Teaching methodology" refers to teaching and learning knowledge and skills, including new learning methods such as PBL, CPX, and simulations. It also includes the skills needed for CPFs and CNIs to understand students' characteristics different from theirs, form a relationship appropriate for practicum education with students, and communicate effectively.

"Partnership" fulfills the need for a "link between school and the clinical field" derived from the definition phase, including reducing the gap between universities and clinical settings and collaboration between CPFs and CNIs from the planning stage of clinical practicum to the evaluation stage to achieve a synergistic effect in practicum education.

\section{Delivery}

The feedback on the developed protocol was positive, as $85 \%-100 \%$ of participants responded that the items and content of the protocol were appropriate. When additional responses on areas of improvement were collected, infection prevention and the need for simulations were emphasized, which were reflected in the final version (Table 1).

\section{DISCUSSION}

Three areas-"concrete guidance and explanation", "providing nursing practice experience", and "link between school and the clinical field"-were derived as participants' needs for pediatric ward practicum instruction. A protocol composed of "detailed information", "teaching methodology", and "partnership" as ways to fulfill these needs was developed.

Graduates and students from nursing schools wanted detailed information about the procedures of the pediatric practicum and detailed explanations about nursing activities from $\mathrm{CPF}$ and CNIs, who first needed practical information about practicum operation and instruction before they could provide information and explanations to students. In previous research [18], CNIs also expressed the desire to receive instructions on basic information, clinical practice policies and procedures, and the curriculum. Therefore, to fulfill the need for "concrete guidance and explanation", "detailed information" was suggested, which included school-based information such as regulations of clinical practicum operation and the practicum schedule, and pediatric ward-based information such as the hospital's clinical practicum policy and the tasks of pediatric ward nurses. An orientation is needed to become an instructor of nursing student practicum in the clinical field [8], and a manual that includes information on the role and responsibilities of faculty, the goals of clinical practice, evaluation criteria, patient characteristics, and the main diagnoses and treatments can be useful [4]. Especially during the COVID-19 pandemic, it is necessary to provide information about prevention guidelines and specific practices.

All participants of this study recognized the reality of the pediatric ward that makes it difficult for students to directly provide care to children due to the risk of infection and felt the need for "providing nursing practice experience" in practicum instruction. Nursing students limit their own activities to avoid interfering with nurses' tasks during the pediatric nursing practicum and only experience simple nursing activities 
Table 1. Elements of the Protocol for Guidance in the Pediatric Nursing Practicum

\begin{tabular}{|c|c|}
\hline Categories & Elements \\
\hline Detailed information & $\begin{array}{l}\text { School-based information } \\
\text { Nursing practice regulations } \\
\text { Role and responsibilities of CPFs and CNIs } \\
\text { Protection of students' right to education } \\
\text { Students' safety standards and legal/ethical issues } \\
\text { Pediatric patient safety standards and legal/ethical issues } \\
\text { Infection prevention strategies and quarantine guidelines } \\
\text { Curriculum (major prerequisite: theory, practice) } \\
\text { Nursing practice objectives and learning outcomes } \\
\text { Syllabus } \\
\text { Practicum schedule and student assignment charts } \\
\text { Clinical practice checklist and core basic nursing skill } \\
\text { Homework assignments } \\
\text { Achievement criteria and evaluation tool } \\
\text { Faculty online site and application } \\
\text { In-school facility and training center information } \\
\text { Related documentation forms } \\
\text { School contact information } \\
\text { Pediatric ward-based information } \\
\text { Hospital policy for students: nursing activities students can perform } \\
\text { Introduction to the pediatric unit: location, structure, treatment and nursing schedule } \\
\text { Pediatric nurses' work and duties } \\
\text { Pediatric patient characteristics: age, common diagnoses, treatment, medication, nursing care } \\
\text { Patient information access and confidentiality: EMR usage } \\
\text { Infection prevention strategies and quarantine guidelines } \\
\text { Pediatric unit contact information }\end{array}$ \\
\hline
\end{tabular}

Teaching methodology

Partnership
Characteristics and needs of today's learners

Communication skills and conflict management

Coping strategies for contextual problems

Critical thinking and PBL

Role of facilitator and feedback

Management and participation of CPX

Management and participation of clinical simulations

Skills for using a high-fidelity simulator

Conference before clinical practice: $\mathrm{CPF}$ and CNI

Sharing of nursing practice schedule and students' assignments

Discussion of syllabus and students' documentation

Decision of pre-learning subjects

Simulation module review

Sharing of teaching schedule

Continued conference: $\mathrm{CPFs}$ and $\mathrm{CNIs}$

Process evaluation

Self-evaluation and improvement plan

Conference after clinical practice: $\mathrm{CPFs}$ and CNIs

Student achievement analysis

$\mathrm{CNI}$, clinical nursing instructor; $\mathrm{CPF}$, clinical practice faculty; $\mathrm{CPX}$, clinical performance examination; EMR, electronic medical record; PBL, problembased learning.

[21]. CPFs also face pressure regarding the operation of the clinical practicum and find it difficult to identify educational practicum sites [22]. As students are unable to provide care to children directly, efforts to supplement or replace direct expe- rience have continued, and various simulation practice techniques $[19,20,22]$ and a mobile education program [23] have been attempted. Since students should learn important nursing skills in a controlled and safe environment, CPFs and 
CNIs must be able to technically operate cases similar to those in the clinical field [24] and utilize high-fidelity simulators. CPFs and CNIs need not only basic information about how to operate the clinical practicum, but also new knowledge and skills about teaching and learning methods [18]. Therefore, the "teaching methodology" item was suggested in developing the practicum instruction protocol, which included detailed information about the operation of simulations and instructions on how to participate. Existing nursing education methods cannot be applied in the circumstances of the COVID-19 pandemic, and COVID-19 has inexorably led to changes in the nursing education system [25]. The instruction and teaching methods for pediatric nursing clinical practice should therefore be updated and improved to reflect those changes. Providing resources to CPFs and CNIs and supporting them to learn new teaching methods and skills will improve nursing competency by enabling nursing students to perform nursing care in a situation similar to that in the clinical field.

To effectively operate practicum education, it is necessary for nursing students, CPFs, and CNIs to have open and fast communication and maintain a constructive relationship [4]. Students in this study expressed the wishes that CNIs would provide detailed information when students started the pediatric practicum, nurses would kindly explain nursing activities even if a student does not ask, and CPFs (i.e., not only $\mathrm{CNIs}$ ) would provide instruction. However, according to a recent study [11], CNIs were bewildered by students' negative attitudes and experienced difficulties due to the lack of support and resources. In order for CPFs and CNIs to instruct students in clinical practice settings, CPFs and CNIs need to understand the characteristics and needs of today's learners, which can be different from theirs [26] and form positive relationships with students. Not only professional competency, but also interpersonal and communication skills, are necessary for effective practicum instruction $[9,10]$. Therefore, the characteristics and needs of today's learners, communication skills, and conflict management were included in "teaching methodology." CPFs and CNIs should receive systematic support to enhance their ability to provide instruction in the practicum through regular training on various teaching methods and their applications.

The CPFs and CNIs in this study expressed the need to communicate and collaborate with each other, starting before the pediatric practicum and extending throughout the duration of the practicum. Nursing students also wanted CPFs to provide instruction together with CNIs in the pediatric ward and wanted their field practicum to be supplemented by simulations in school. Therefore, to fulfill the need for a "link between school and the clinical field", "partnership" was sug- gested, which included discussions and collaborations between CPFs and CNIs from before clinical practice to the end. In order for clinical practicum to be operated effectively and improved, the cooperation structure between the academic institution and the practicum site is important [7]. A previous study [27] suggested the academic practice partnership model as a revolutionary method for the education of nurse practitioners. To provide instruction on practice in the clinical field, efforts to reduce the gap between theory and practice and to promote collaboration between academic and practicum departments are needed [4]. Especially during the COVID-19 pandemic, $\mathrm{CPFs}$ and CNIs should share information about changes in pandemic-related circumstances and must work together to make the best possible decisions after closely discussing the corresponding changes in the operation of students' clinical practicum and practicum instruction.

The protocol developed in this study will help with the operation of high-quality practicum education and contribute to the improvement of nursing students' pediatric nursing practice competencies by being utilized to facilitate pediatric practicum instruction by CPFs and CNIs. This study included common content in the pediatric nursing practicum instruction protocol so that CPFs and CNIs can both use it. However, the context of practicum instruction and the needs of CPFs and CNIs can be different. In the future, it will be necessary to develop separate practicum instruction guidelines and support programs for $\mathrm{CPFs}$ and $\mathrm{CNIs}$.

\section{CONCLUSION}

$\mathrm{CPFs}$ and CNIs play important roles in the pediatric practicum for nursing students, and the COVID-19 pandemic has led to changes in the method of practicum instruction, in response to which improvements need to be made. This study aimed to develop a protocol on pediatric ward practicum instruction that can provide CPFs and CNIs with guidelines for pediatric nursing practicum instruction. Using the double diamond process with the discovery, definition, development, and delivery phases, this study identified the need for concrete guidance and explanation, providing nursing practice experience, and a link between school and the clinical field. A protocol comprising items including detailed information, teaching methodology, and partnership as methods to fulfill these needs was developed. The protocol developed in this study will enhance nursing students' pediatric nursing practice competencies by being utilized in pediatric practicum instruction by CPFs and CNIs. The protocol will also help operate high-quality practicum education and contribute to increasing the level of practicum education. 


\section{ORCID}

Hyun Young Koo

https:// orcid.org/0000-0001-5848-2143

Bo Ryeong Lee

https://orcid.org/0000-0003-4014-9056

\section{Authors' contribution}

Conceptualization: Hyun Young Koo; Data collection, Formal analysis: all authors; Writing-original draft: Hyun Young Koo; Writing-review and editing: all authors; Final approval of published version: all authors.

\section{Conflict of interest}

Hyun Young Koo has been the editor-in-chief of Child Health Nursing Research from January 2018 to December 2021, but had no role in the decision to publish this manuscript. Otherwise, no existing or potential conflict of interest relevant to this article was reported.

\section{Funding}

This study was supported by the research fund of Daegu Catholic University in 2020.

\section{Data availability}

Please contact the corresponding author for data availability.

\section{Acknowledgements}

None.

\section{REFERENCES}

1. Korean Society of Nursing Science. Learning objectives of subjects for nursing students. Seoul: Korean Society of Nursing Science; 2017.

2. Ulenaers D, Grosemans J, Schrooten W, Bergs J. Clinical placement experience of nursing students during the COVID-19 pandemic: a cross-sectional study. Nurse Education Today. 2021;99:104746. https://doi.org/10.1016/j.nedt.2021.104746

3. Korean Accreditation Board of Nursing Education. The manual of nursing education accreditation for university. Seoul: Korean Accreditation Board of Nursing Education; 2021. p. 58-59, 143-145.

4. Stevens KE, Duffy EA. A toolkit for nursing clinical instructors. Teaching and Learning in Nursing. 2017;12(2):170-172. https://doi.org/10.1016/j.teln.2016.04.003

5. Song MR, Kim IK, Kim YK. Experience of clinical practice faculty and nurses on clinical nursing practicum for nursing students.
Qualitative Research. 2010;11(2):106-118.

6. Park KO, Kim JK, Yu M. Experience of role adaptation from nurse to member of the nursing faculty. Journal of Korean Academy of Nursing. 2016;46(2):168-182.

https://doi.org/10.4040/jkan.2016.46.2.168

7. Chung MS, Park JS, Ryu E, Shin G, Jun HY, Kim BJ. Teaching effectiveness and adequacy of practical training in nursing students. Journal of Korean Academic Society of Nursing Education. 2015; 21(4):550-560. https://doi.org/10.5977/jkasne.2015.21.4.550

8. Brown SM. Cultivating adjunct clinical nursing instructors for clinical education. Maryland Nurse. 2019;20(2):12.

9. Park I, Suh YO. Development of teaching efficacy scale to evaluate clinical nursing instructors. Korean Journal of Adult Nursing. 2018;30(1):18-29. https://doi.org/10.7475/kjan.2018.30.1.18

10. Collier AD. Characteristics of an effective nursing clinical instructor: the state of the science. Journal of Clinical Nursing. 2018;27(1-2): 363-374. https://doi.org/10.1111/jocn.13931

11. Park YA, Kong EH, Park YJ. Head nurses' experiences in clinical practice education of nursing students: a qualitative research. Journal of Korean Academic Society of Nursing Education. 2018;24(4): 337-346. https://doi.org/10.5977/jkasne.2018.24.4.337

12. Design Council. Video: what is service design? [Internet]. London: Design Council; 2015 [cited 2020 May 1]. Available from:

https://www.designcouncil.org.uk/news-opinion/video-whatse rvice-design

13. Design Council. What is the framework for innovation? Design Council's evolved Double Diamond [Internet]. London: Design Council; 2015 [cited 2020 May 1]. Available from:

https://www.designcouncil.org.uk/news-opinion/design-proce ss-what-double-diamond

14. Kwon S, Nah K. A Study on improvements of space allocation for small and mediumsized orthopedic hospitals: applying the service design methodology-focused on remodeling scheme of Yeosu- Baek Hospital. Journal of Korea Design Knowledge. 2014;32:375-386. https://doi.org/10.17246/jkdk.2014..32.034

15. Cha H, Hwang Y, Noh E. Development and verification of user-centered design guidelines on online support system for curriculum at primary/secondary schools. Journal of The Korean Association of Information Education. 2021;25(3):511-525. https://doi.org/10.14352/jkaie.2021.25.3.511

16. Cho JH. A study of developing children safety education program in theatres: focused on utilizing service design. Korean Journal of Arts Education. 2016;14(3):253-276.

17. Elo $S$, Kyngäs $H$. The qualitative content analysis process. Journal of Advanced Nursing. 2008;62(1):107-115. https://doi.org/10.1111/j.1365-2648.2007.04569.x

18. Davidson KM, Rourke L. Surveying the orientation learning needs of clinical nursing instructors. International Journal of Nursing Education Scholarship. 2012;9(1):Aticle 3. https://doi.org/10.1515/1548-923X.2314 
19. Fonseca LM, Aredes ND, Fernandes AM, Batalha LM, Apóstolo JM, Martins JC, et al. Computer and laboratory simulation in the teaching of neonatal nursing: innovation and impact on learning. Revista Latino-Americana de Enfermagem. 2016;24:e2808. https://doi.org/10.1590/1518-8345.1005.2808

20. Park SN, Im YS. Utilizing video vs simulation practice for handoff education of nursing students in pediatric nursing. Child Health Nursing Research. 2018;24(1):27-36.

https://doi.org/10.4094/chnr.2018.24.1.27

21. Choi EA, Lee KE, Lee YE. Nursing students' practice experience in neonatal intensive care units. Child Health Nursing Research. 2015;21(3):261-271. https://doi.org/10.4094/chnr.2015.21.3.261

22. Kim SG. Effects of a simulation-based high-risk neonatal care education on learning satisfaction, class participation, learning motivation and clinical competency in nursing students. Journal of the Korea Academia-Industrial cooperation Society. 2015;16(10):6807-6815. https://doi.org/10.5762/KAIS.2015.16.10.6807

23. Seo HK. Development and effect of a mobile education program for nursing students on high-risk infant. Journal of Korean Society for Simulation in Nursing. 2019;7(2):71-82.

https://doi.org/10.17333/JKSSN.2019.7.2.71

24. Parsh B. Characteristics of effective simulated clinical experience instructors: interviews with undergraduate nursing students. Journal of Nursing Education. 2010;49(10):569-572. https://doi.org/10.3928/01484834-20100730-04

25. Haslam MB. What might COVID-19 have taught us about the delivery of nurse education, in a post-COVID-19 world? Nurse Education Today. 2021;97:104707.

https://doi.org/10.1016/j.nedt.2020.104707

26. Kowalski K, Horner M, Carroll K, Center D, Foss K, Jarrett S, et al. Nursing clinical faculty revisited: the benefits of developing staff nurses as clinical scholars. Journal of Continuing Education in Nursing. 2007;38(2):69-75. https://doi.org/10.3928/00220124-20070301-08

27. LeFlore JL, Thomas PE. Educational changes to support advanced practice nursing education. Journal of Perinatal and Neonatal Nursing. 2016;30(3):187-190.

https://doi.org/10.1097/JPN.0000000000000201 